\title{
The Design of Car Controlled by Internet of Things Based on Microcontrollers Cortex-M3
}

\author{
Tang Lili, ${ }^{1, a}$, Huang Wei ${ }^{2, b}$ \\ ${ }^{1}$ Department of Computer \& Information Engineering, Zhixing College of Hubei University, Wuhan, \\ 430011, China \\ ${ }^{2}$ System Design Institute of Hubei Aerospace Technology Academy, Wuhan, 430040, China \\ aemail: toney2001@126.com, bemail:jijunhuang@126.com
}

Keyword: Internet of Things; LPC1768; Wifi; remote monitoring

\begin{abstract}
This design uses Wifi control the movement of the car, the speed acquisition, temperature acquisition and monitoring of video image. The car controller uses microcontroller LPC1768, which is 32-bit, single cycle, support reduced instruction set and has rich resources of peripherals and high-speed processing speed. Using computer PC software VC6.0, through WinSocket programming, a wireless router and the car's Wifi serial interface module form a wireless local area network to realize controlling the movement of the car, displaying the speed and surrounding temperature of the car, and acquiring the car camera on the computer. This design can be used in remote monitoring, industrial robots, intelligent household, unmanned driving and other fields,which has a wide range of application areas and certain market value in use.
\end{abstract}

\section{Brief Introduction of the Function of Car Controlled by Internet of Things}

Car controlled by internet of things connects computer PC with car through Wifi network, meanwhile the meaning of internet of things mainly means that there are lots of sensors on the car, which utilize wifi and host computer to interact car's data of speed, temperature and camera etc. through the platform of car. And that's the connotation of car controlled by internet of things. The concretely implemented function is that using PC host computer and WLAN to make computer, wireless router and car in network, whose mode includes ad-hoc network and basic network. Users can manipulate the car's movement to get the collected information by controlling computer PC within the local area network. If possessing a fixed IP, remote control can also be realized. Car controlled by internet of things's topology is shown in figure 1.

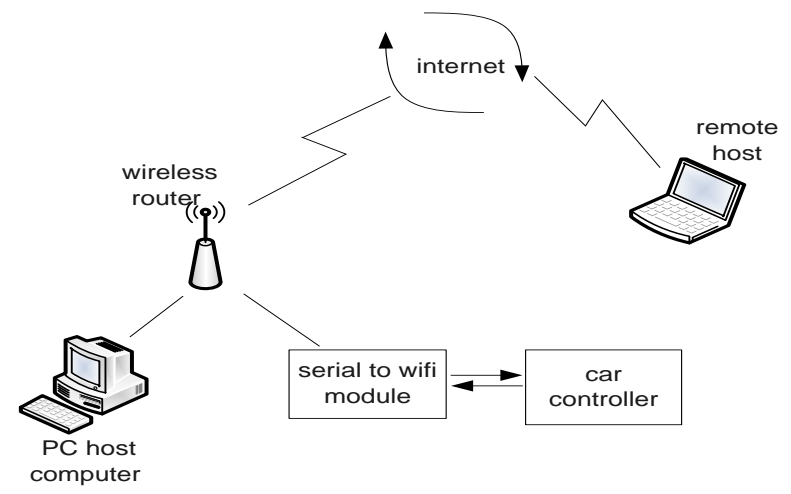

Fig.1. Topology of Car Controlled by Wifi Internet of Things

Connecting computer software, wireless router, and the car by forming ad-hoc network or basic net, and if there's a fixed IP, the car can also be connected with remote server through internet technology, which can also realize surpassingly remote control. The PC software compiles internet network interface mainly by winsocket programming, the car side converts wifi signals into serial signals via converting uart module into wifi module, and then sends wifi signals to connect car and PC software by wireless router, and this kind of mode is basic net. Another kind of networking mode is ad-hoc network, which directly sends wifi signals by converting wifi module into serial 
communications module, then it receives signals by means of the computer's WLAN card. Ad-hoc network can also realize network communication, but can’t realize remote connection.

\section{The Introduction of NXP LPC1758 Chip}

The Cortex-M3 microcontroller of LPC1700 series is a latest MCU with high performance and low power consumption which is launched by NXP company. Its peripheral components include a flash memory up to $512 \mathrm{~KB}$, a $64 \mathrm{~KB}$ data memory, Ethernet MAC, USB host/slave/OTG interface, 8 channel generic DMA controller, 4 UART, 2 CAN channel, 2 SSP controller, SPI interface, 3 I2C interface, a 2-input and 2-output I2S interface, 8-channel 12-bit ADC, 10-bit DAC, motor control PWM, quadrature encoder interface, 4 GP Timer, 6 - output general PWM, ultra-low power consumption RTC with separate battery power and general I/O pins as many as $70[1]$.

\section{The Design of PC}

The main function of PC is to set the car's relevant parameters as well as acquire and show the relevant information collected by car through TCP/IP network communication protocols and controlling the car's operation [2]. PC adopt VC6.0 integrated development environment earlier launched by Microsoft. This design mainly includes the following aspects of programming:

(1)TCP/IP network communication protocols and interface;

(2)The control region of car movement;

(3)The speed dial of car;

(4)The car's temperature display meter;

(5)The car's surveillance video display area (extended content).

\section{The Design of Lower Computer}

Lower computer, namely the car's controller, controls all of the car's actions. Its main contents is included as follows:

(1)The drive converting Wifi into serial port [3];

(2)The car movement control;

(3)The drive of DS18B20 temperature sensor;

(4)The acquisition of car speed;

(5)Parsing and encapsulation of communication data. 


\section{The flow chart of module design for lower computer}

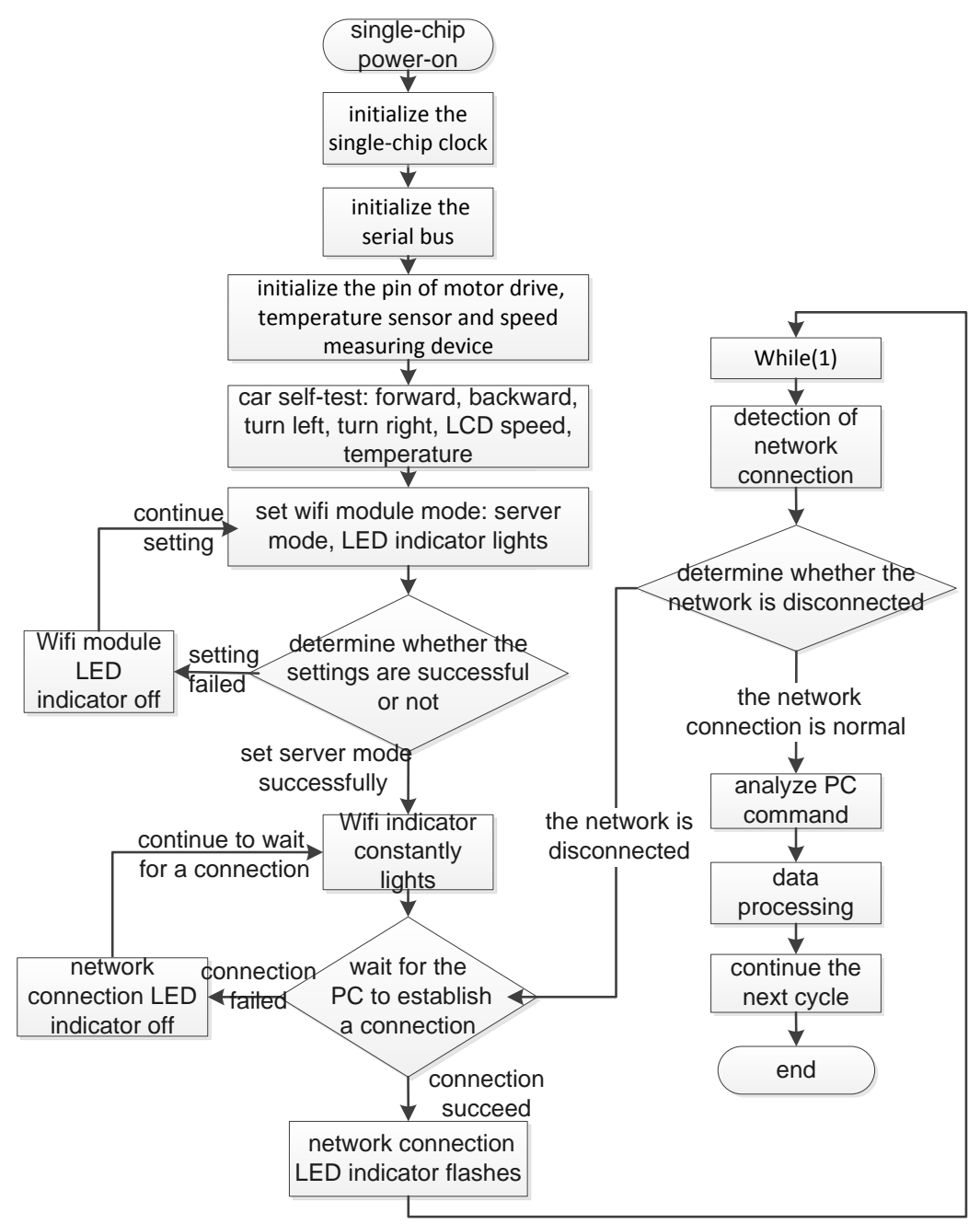

Fig.2. The flow chart of design for lower computer

\section{Serial design of lower computer}

Lower machine serial design mainly involves initialization of serial ports, serial ports sending, serial ports reception and functions of serial ports interruption [4] [5]. MCU realizes communications via serial port and converting Wifi into serial port module, which is the communication part of car controlled by internet of things. LPC1758 MCU includes 4 serial ports, and possesses FIFO first-in first-out buffer and various serial ports interruption which can receive more than one bit at the same time. Via setting relevant register, it can well realize communication function. MCU receives and sends instructions to PC via controlling communications between serial port and PC software, thus the car's corresponding action can be controlled via analyzing the instructions sent by PC.

\section{The car control program for lower computer}

void MotorCtrl(bit leftM, bit leftM_UP, bit rightM, bit rightM_UP, uint speed)

\{//F_:Motor switch

EN1=leftM;

EN2=rightM;

scale $=$ speed;

//F_: forward 


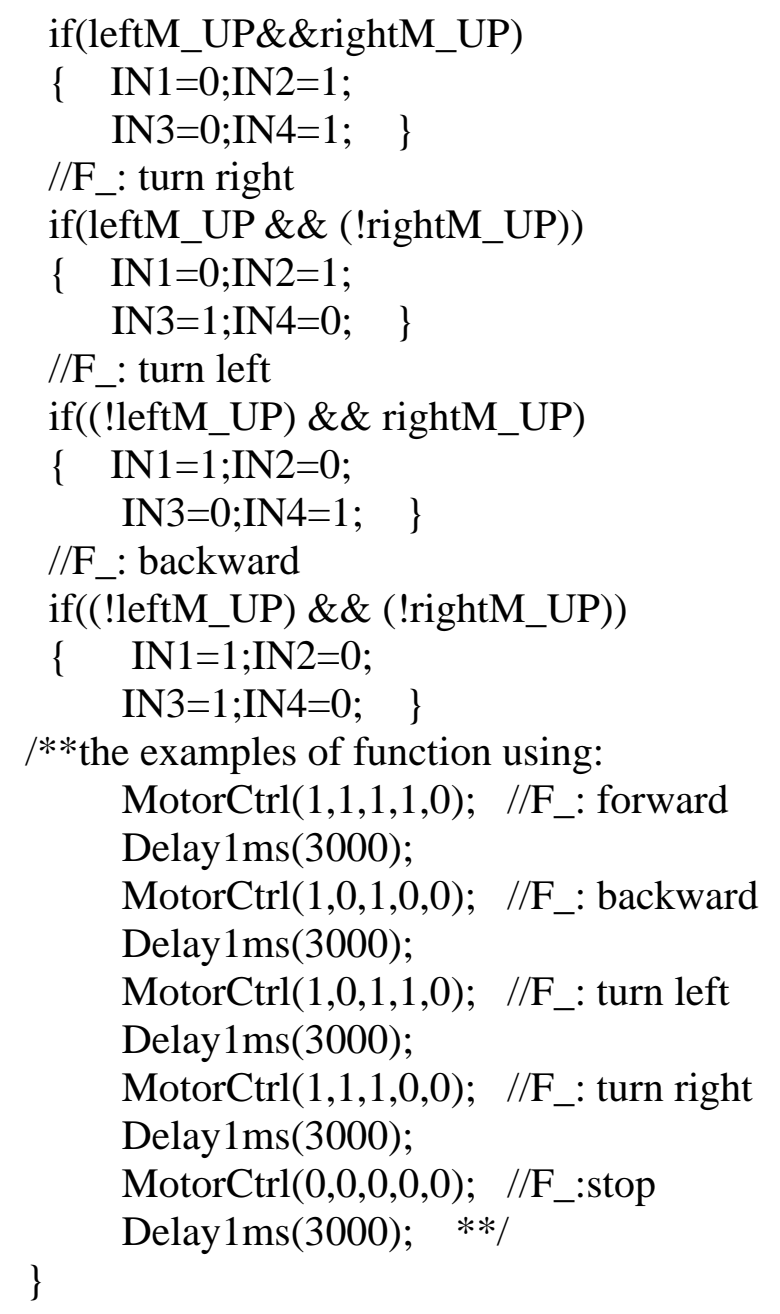

\section{Conclusions}

Through debugging and modifying codes time after time, the project is basically formed, and can achieve expected function. The communication is stable, the car's control is reliable, the accuracy and sensitivity of data acquisition meets requirements. The tests which is done on the occasion that has nothing to do with the remote network control prove that stability and reliability of the system has already far exceeded the ordinary infrared remote control cars and wireless RF remote control cars. The significance of this design lies in efficiently combining Computer software technology, network technology and MCU technology. This design also highlights and deepen the implication of "internet of things", so in the future, it must be widely used in social activities and manufacture.

\section{References}

[1] ARM Limited. Cortex-M3 Technical Reference Manual.2006.

[2] Genshen Cui, Anqing Sun. ARM embedded system development and practice [M]. Beijing: China power press, 2008.

[3] Yao Chen. The Cortex-M3+ $\mu \mathrm{C} / \mathrm{OS}-\mathrm{II}$ introduction to embedded system development and application [M]. Beijing: people's posts and telecommunications publishing house, 2010.

[4] Zhaoqian Qie. Embedded system hardware system design [M]. Beijing: university of aeronautics and astronautics press, 2007.

[5] Zhaoqian Qie. Embedded graphical system design [M]. Beijing: university of aeronautics and astronautics press, 2009. 